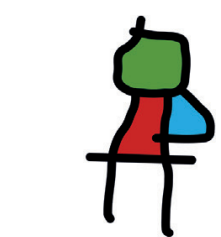

SEICAIP

\section{Allergologia et immunopathologia}

Sociedad Española de Inmunología Clínica, Alergología y Asma Pediátrica

www.all-imm.com

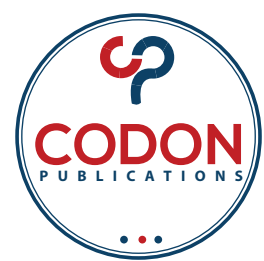

ORIGINAL ARTICLE

OPEN ACCESS (C)(1)(2)

\title{
Atypical food protein-induced enterocolitis syndrome in children: Is IgE sensitisation an issue longitudinally?
}

\author{
Athina Papadopouloua*, Theano Lagousi ${ }^{\mathrm{b}}$, Elpiniki Hatzopoulou ${ }^{\mathrm{b}}$, Paraskevi Korovessia ${ }^{\mathrm{a}}$, \\ Stavroula Kostaridou ${ }^{b}$, Despina -Zoe Mermiri ${ }^{\mathrm{a}}$
}

\begin{abstract}
${ }^{a}$ Department of Allergology and Pulmonology, Penteli's Children's Hospital, Athens, Greece
${ }^{b}$ Department of Pediatrics, Penteli's Children's Hospital, Athens, Greece
\end{abstract}

Received 25 October 2020; Accepted 22 November 2020

Available online 1 May 2021

\section{KEYWORDS \\ atypical food protein-induced enterocolitis syndrome; food allergy; Immunoglobulin E; long-term outcomes; asthma}

\begin{abstract}
Background: Food Protein-Induced Enterocolitis Syndrome (FPIES) is a clinically well-characterised, non-Immunoglobulin E (IgE)-mediated food allergy syndrome, yet its rare atypical presentation remains poorly understood.

Objective: Aim of this study was to present the 10-year experience of a referral centre highlighting the atypical FPIES cases and their long-term outcome.

Methods: FPIES cases were prospectively evaluated longitudinally in respect of food outgrowth and developing other allergic diseases with or without concomitant IgE sensitisation.

Results: One hundred subjects out of a total of 14,188 referrals $(0.7 \%)$ were identified. At presentation, 15 patients were found sensitised to the offending food. Fish was the most frequent eliciting food, followed by cow's milk and egg. Tolerance acquisition was earlier for cow's milk, followed by egg and fish, while found not to be protracted in atypical cases. Resolution was not achieved in half of the fish subjects during the 10-year follow-up time. Sensitisation to food was not related to infantile eczema or culprit food, but was related to sensitisation to aeroallergens. In the long-term evaluation, persistence of the FPIES or aeroallergen sensitisation was significantly associated with an increased hazard risk of developing early asthma symptoms. Conclusion: Sensitisation to food was related neither to eczema or culprit food nor to tolerance acquisition but rather to the development of allergic asthma through aeroallergen sensitisation. In addition to an IgE profile at an early age, FPIES persistence may also trigger mechanisms switching FPIES cases to a T-helper 2 cells immune response later in life, predisposing to atopic respiratory symptoms; albeit further research is required.

(C) 2021 Codon Publications. Published by Codon Publications.
\end{abstract}

*Corresponding author: Dr Athina Papadopoulou, MD, Pediatric EAACI Allergist, Allergology and Pulmonology Unit, Penteli Children Hospital, Athens, Greece. E-mail address: athinapap@yahoo.com 


\section{Introduction}

Food Protein-Induced Enterocolitis Syndrome (FPIES) is a non-Immunoglobulin E (IgE)-mediated, clinically well-characterised food allergy syndrome, yet its rare atypical presentations remain poorly understood even in recently published International consensus guidelines.,2 The term 'atypical FPIES' was first coined by Sicherer et al in $1998 .^{3}$ Nowadays, it is considered for subjects presenting with a clinical history consistent with FPIES, and also demonstrating allergic sensitisation to the offending food. ${ }^{4-6}$

Food protein-induced enterocolitis syndrome is mainly classified as a non-lgE-mediated disorder with underlying distinct pathophysiological, local and systemic immunological reactions, characterised by an increased production of TNF- $\alpha$, profound activation of innate immune cells and inadequate secretion of TGF- $\beta$ and food-specific IgA. ${ }^{7-10}$ Moreover, it has been shown that in cow's milk FPIES, both humoral and cellular-specific immune responses were weak or absent. ${ }^{11,12}$ The authors reported significantly low total IgE, serum food-specific IgG4 and low secretion of regulatory cytokine IL-10. On the other hand, a single case was reported with high levels of IL-4 and IL-5 cytokines and a decrease expression in IFN- $\gamma$ in peripheral $\mathrm{T}$ cells during acute non-IgE FPIES, implying a T-helper 2 cells (Th2)skewed response. ${ }^{13}$ Additionally, there were few patients reporting a typical association with an IgE-mediated mechanism against the culprit food linking it to a more protracted course of the disease. , $^{2,14-17}$ Moreover, it has been postulated that local production of mast cells could facilitate antigen uptake, promoting diffused colitis. ${ }^{18}$

It remains unknown whether or not the concomitant IgE sensitisation constitutes a diverse FPIES phenotype with distinct clinical characteristics and outcome pathways. ${ }^{6}$ Moreover, it is not clear whether these subjects follow a similar pattern with other atopic diseases regarding the so-called Allergic March model, albeit a recent review highlighted observations linking persistent non-lgE-mediated food allergy to the emergence of IgE-mediated comorbidities (rhinitis or asthma). ${ }^{19}$ This study was conducted aiming at assessing atypical clinical presentations and long-term outcomes of FPIES cases with or without concomitant IgE sensitisation and proposing a possible non-lgE allergy march process.

\section{Materials and Methods}

\section{Patient identification and food challenge protocol}

Acute and chronic FPIES are defined based on NowakWęgrzyn et al criteria. ${ }^{2}$ FPIES patients, referred from central and southern Greece to the Allergology and Pulmonology Department, were enrolled during a 10-year period, from January 2008 to December 2017.

Patients' data (age, gender, implicated food, disease onset, clinical presentation, self-reported atopic family history and eczema) were recorded at baseline. IgE food sensitisation was evaluated at diagnosis through skin prick tests (SPTs) and specific IgE determination using commercial extracts (ALK-Abello, Horsholm, Denmark and RAST,
Pharmacia Diagnostics AB, Uppsala, Sweden). SPTs included cow's milk, egg (yolk and white), fish, nuts, cereals and molluscs. A serum-specific IgE value higher than $0.35 \mathrm{KU} / \mathrm{L}$ or an SPT wheal diameter of $>3 \mathrm{~mm}$ was considered positive. Oral food challenge (OFC) was performed to confirm the diagnosis in inconclusive FPIES cases and to evaluate tolerance according to the consensus protocol. ${ }^{2}$ OFCrelated data were collected.

\section{Age of resolution andlong term follow up}

All patients were followed up prospectively from presentation to June 2019. Patients were evaluated at six-month intervals (clinical assessment, other atopic symptoms, accidental contact with the culprit food and possible reactions). Consumption of dairy products or eggs (boiled or baked) was recorded. Age of tolerance was recorded by either self-resolution or OFC with full home re-introduction. Specific mention was made of cow's milk, egg and fish resolution age in relation to lgE profile.

Moreover, all patients were followed up prospectively in terms of developing asthma. Current respiratory symptoms were considered if present during the last year's follow-up evaluation. Spirometry, SPTs and specific IgE (slgE) to aeroallergens (Dermatophagoides, Pteronyssinus, Dermatophagoides Farinae, Alternaria Alternata, Bermuda, Meadow Fescue and Timothy grass, Olive, Parietaria Judaica and Officinalis) were performed guided by symptomatology.

The study protocol was approved by the Institutional Review Board of our children's hospital, and written informed consent was obtained from the guardians of all subjects.

\section{Statistical Analysis}

This is a prospective, descriptive, longitudinal study. As 100 patients were finally evaluated, when referring to the total FPIES cases, real numbers were used that also represented the corresponding percentage; in all the other cases, percentages were applied. The normality of our data was assessed by the Kolmogorov-Smirnov test, and corresponding 95\% confidence intervals $(\mathrm{Cl})$ were recorded. Descriptive analysis was used to calculate central tendency and dispersion of continuous variable using the Mann-Whitney $U$ test and the Kruskal-Wallis test for non-parametric independent samples, whereas Chi-square test and risk coefficients using Pearson's $r$ or Spearman's $\rho$ were used to compare nominal variables. Bivariate data were evaluated according to the type of clinical presentation (acute or chronic) and the presence or absence of IgE mechanism and atopic comorbidities. The Kaplan-Meier survival analysis was applied as a time-to-event method to estimate the age probability of outgrowing FPIES in various foods and its relation to atopic background. Log-rank test was used to compare median time of tolerance in different clinical subgroups of FPIES cases. The Cox regression analysis was used to evaluate the proportionality of tolerance (PT) ratio $(95 \% \mathrm{Cl})$ between subjects with different foods or atopic history. If $\mathrm{PT}=1$, no relation between the factors was found; if $\mathrm{PT}>1$, a positive influence for earlier FPIES 
outgrowth was detected; and if $\mathrm{PT}<1$, a negative effect was reported. IgE sensitisation to food or aeroallergens was further analysed using multiple regression to study correlation with respiratory allergic symptoms. Moreover, the Kaplan-Meier analysis and hazard function were also applied to evaluate the longitudinal effect of sensitisation on the development of respiratory symptoms. Statistical significance was set at $\mathrm{P}<0.05$. SPSS V20.0 (IBM, Chicago, IL, USA) was used for statistical analysis.

\section{Results}

\section{FPIES overview and atopy signs at presentation}

Overall, 100 confirmed FPIES cases $(0.7 \%)$ out of a total of 14,888 referred for various atopic conditions were included. Nine more subjects were excluded, given the inability to confirm the diagnosis or a shorter than 24-month follow-up (Figure 1a). No significant gender predominance was observed (55 males). Fish was the most frequently triggering food, followed by cow's milk, egg, chicken and shrimp. The most common fish involved was cod, while perch, sardine, tope and sea bream were responsible for one case each. Among FPIES cases related to egg, one was caused exclusively by egg yolk. The vast majority of patients were acute (89 cases). Eleven patients were chronic, all reacting to cow's milk. Vomiting was the predominant symptom in 90 FPIES patients (Figure 1b). Chronic patients mainly presented with diarrhoea and moderate to severe gastroesophageal reflux. Vomiting was not the main presenting symptom in these patients. Only two patients showed failure to thrive whose diagnosis was delayed. Two infants presented with episodes of acute urticaria after consuming cow's milk during the breastfeeding period, yet having no IgE positive test. All these patients with inconclusive history had late onset of vomiting when OFC was performed to confirm the diagnosis. Among acute FPIES cases, 33 required admission, mainly for fluid resuscitation or lethargy. Milk FPIES was significantly related to hospitalisation
(OR, 95\% Cl: 2.75, 1.11-6.80). The mean age at appearance of symptoms was $9.8 \pm 7.4$ months, depending on feeding practices and the culprit food. Thus, the mean age for cow'smilk, egg and fish FPIES cases was $2.8 \pm 2.4,11 \pm 5.9$ and $13 \pm 6.7$ months, respectively.

IgE sensitisation to offending food was observed in 15 of the total 100 FPIES cases (15\%). In all, $16.12 \%$ of fish FPIES and $17.8 \%$ of cow's milk FPIES cases were sensitised. There was no significant relation between food sensitisation and type of FPIES (acute or chronic) (OR, 95\% Cl: 1.19, 0.23-6.18). Neither history of eczema (OR, 95\% Cl: 2.50, $0.68-9.68)$ nor atopy in the family $(\mathrm{OR}, 95 \% \mathrm{Cl}: 1.0,0.59$ 1.59) was related to sensitisation.

\section{FPIES outcome}

The mean follow-up period was $92 \pm 54.4$ months, ranging from 24 to 144 months. Six fish FPIES patients denied challenge, expressing fear, and four others were lost to follow-up. There was no significant difference in clinical and laboratory characteristics of these patients compared with other patients. Finally, OFC was performed in 82 patients to confirm or refute tolerance acquisition, while eight subjects self-reported resolution. No patient aged less than 12 months was challenged. A total of 55 patients acquired tolerance to the offending food, while OFC was positive in 35 patients. The median age of tolerance acquisition for all patients was 78 months (62-93 months). For the total number of subjects, tolerance rate was $44 \%$ and $69 \%$ at 5 and 10 years of follow-up, respectively (Figure $2 \mathrm{a}$ ). Proportionality of tolerance was not correlated to the presence of eczema or family history of atopy (Table 1). Moreover, PT was not related to lgE sensitisation of the offending food (Table 1, Figure 2b).

\section{Milk}

All patients with cow's milk FPIES have successfully tolerated milk at a median age of 20 months (Figure 2c). Interestingly, three non-lgE subjects had a positive

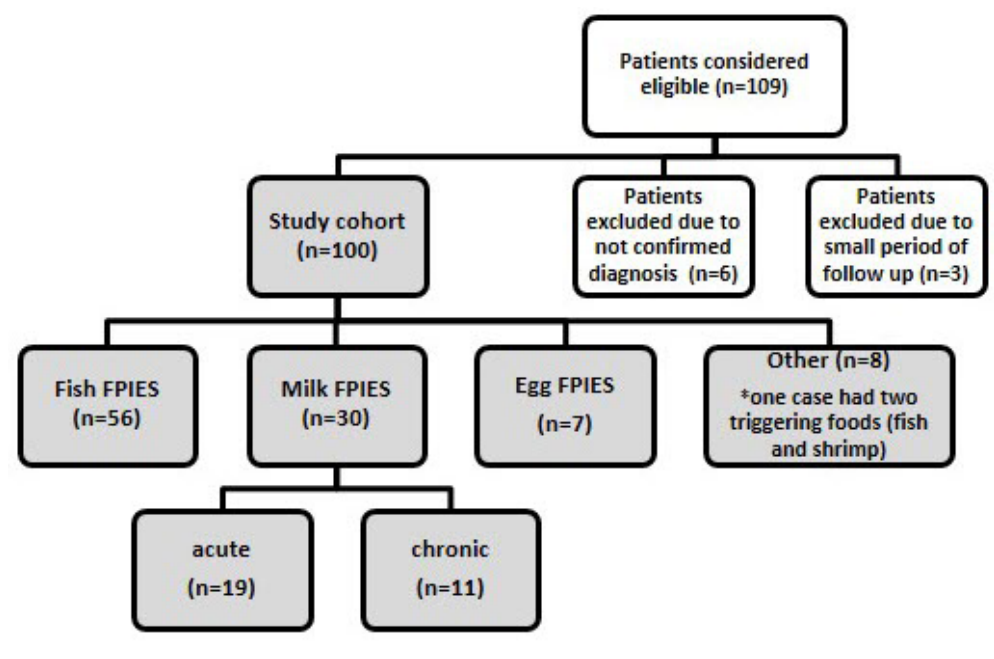

(a)

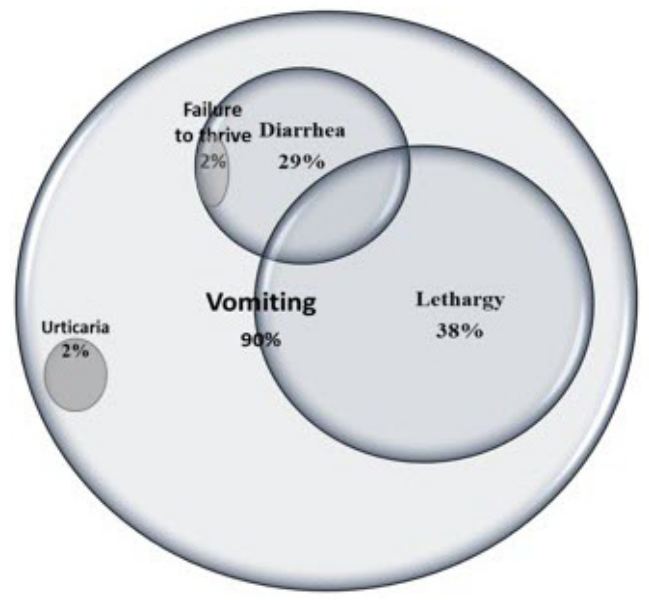

(b)

Figure 1 (a) Flow-chart of study population. (b) Presenting symptoms in food protein-induced enterocolitis syndrome (FPIES) cases. 


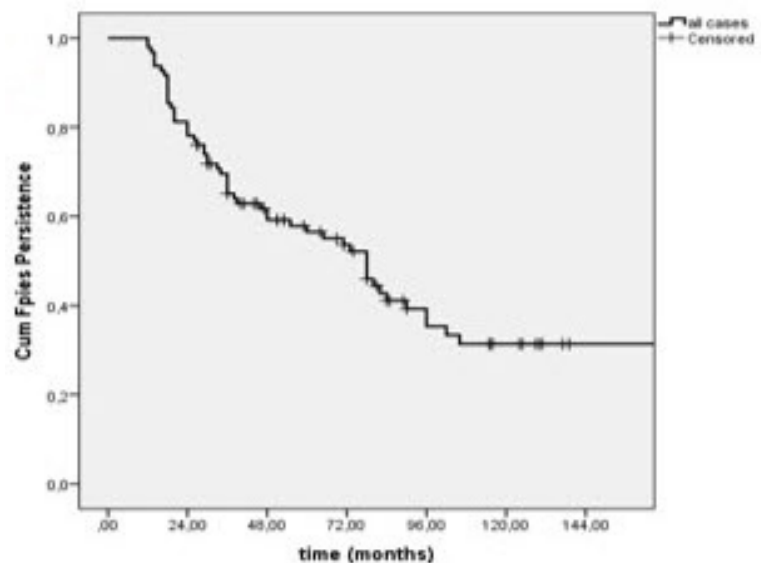

(a)

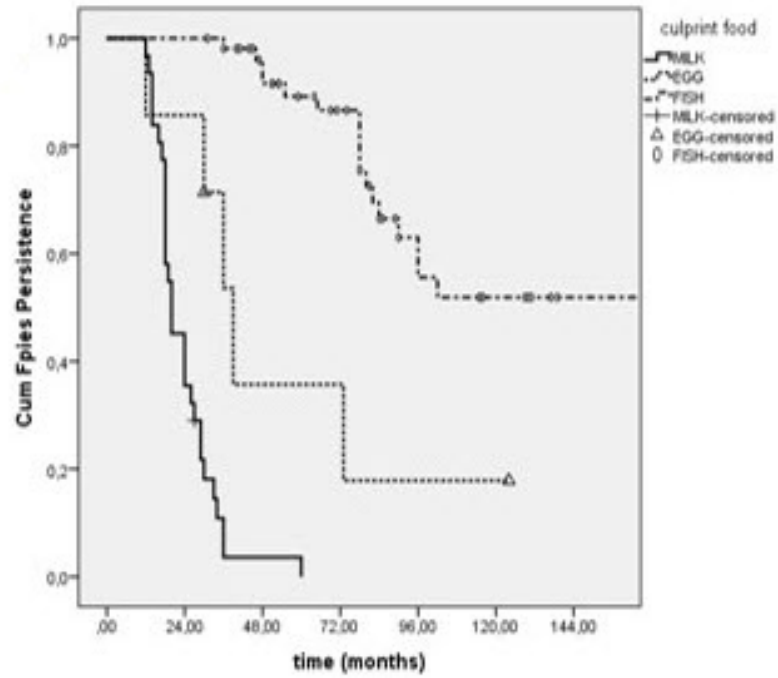

(c)

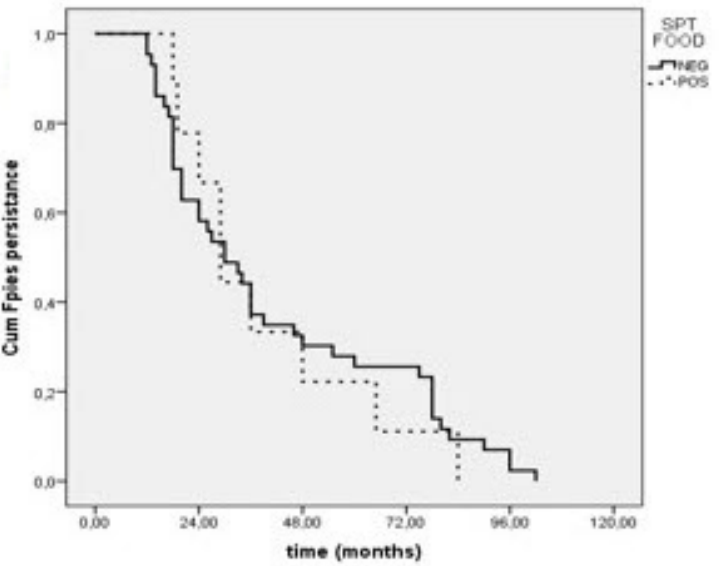

(b)

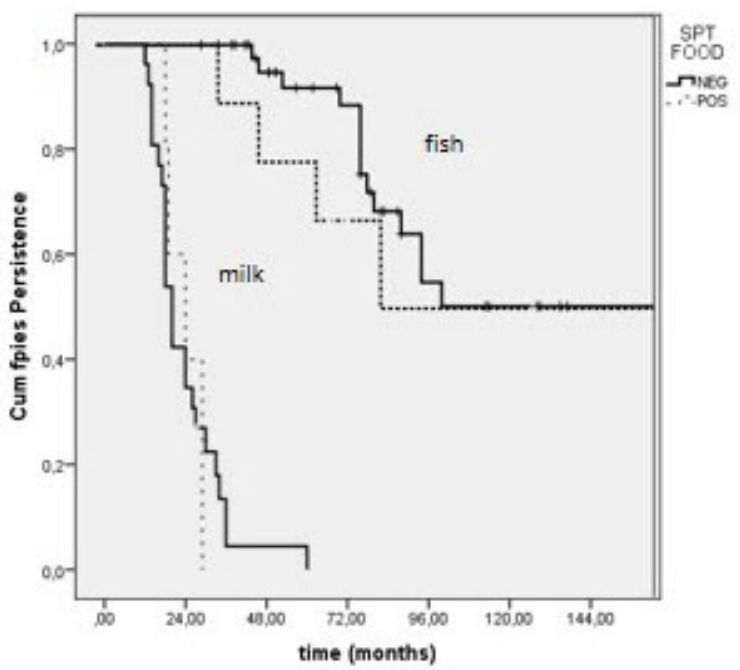

(d)

Figure 2 The Kaplan-Meier functions showing the persistence of food protein-induced enterocolitis syndrome (FPIES) according to (a) total cases, (b) IgE to food, (c) the culprit food and (d) culprit food and IgE to food.

Table 1 The cox regression proportional model analysing the proportional tolerance (PT) ratio in FPIES cases in terms of different parameters (listed in the first row).

\begin{tabular}{lcccc}
\hline & Coefficient & PT & $95 \% \mathrm{Cl}$ & $\mathrm{P}$ \\
\hline Family history of atopy & -0.45 & 0.63 & $0.32-1.24$ & 0.18 \\
Ever eczema & -0.65 & 0.51 & $0.20-1.33$ & 0.17 \\
Sensitisation to food & 0.23 & 1.26 & $0.52-3.06$ & 0.59 \\
Sensitisation to & 0.09 & 0.88 & $1.09-0.31$ & 0.88 \\
$\quad$ aeroallergens & & & & \\
Offending food (fish) & -1.56 & 0.20 & $0.12-0.35$ & 0.001 \\
\hline
\end{tabular}

FPIES: food protein-induced enterocolitis syndrome.

challenge to raw milk but were able to consume other milk products, such as yogurt or cheese, uneventfully. These patients were considered to have outgrown FPIES as they could consume processed milk products. No significant difference in median tolerance time was recorded neither between chronic and acute nor between IgE and non-IgE milk-sensitised FPIES patients $(\mathrm{P}=0.64$ and 0.89 , respectively; Figure 2d).

Fish

Fish FPIES patients showed a significant delay in resolution compared to other FPIES cases (Table 1). Only $11 \%$ and $48 \%$ of patients successfully passed the OFC by 5 and 10 years, respectively (Figure $2 \mathrm{c}$ ). The median age of a successful fish OFC was estimated to be 115 months. Although there was a difference in achieving tolerance in the first six years, no significant difference was found in the median age of tolerance acquisition between food-sensitised and food non-sensitised patients for the total follow-up time $(P=0.43$, Figure $2 d)$.

\section{Egg}

Seven (all) patients with egg FPIES completed an OFC, five of these uneventfully. The median age of a successful egg 
Table 2 P-values and OR $(95 \% \mathrm{Cl})$ comparing atopy syndromes with the offending food, IgE sensitisation and family history of atopy in FPIES cases. Sensitisation to food allergens was related with sensitisation to aeroallergens. Family history of atopy and fish FPIES were related with asthma and sensitisation to aeroallergens. Statistical significance was set at $\mathrm{P}<0.05$.

\begin{tabular}{lllll}
\hline & \multicolumn{1}{c}{ Eczema } & Ever asthma & IgE to food & IgE to aeroallergens \\
\hline Offending food & & & & 15 \\
$\quad$ All & 16 & 25 & 10 & 14 \\
$\quad$ Fish & 13 & 22 & 5 & 13 \\
$\quad$ Milk & 3 & 2 & 0 & 0 \\
$\quad$ Egg & 0 & 1 & 0.64 & 0.03 \\
P & 0.15 & 0.004 & $1.38(0.48-1.56)$ & $1.15(1.07-9.17)$ \\
OR $(95 \% \mathrm{Cl})$ & $2.85(0.74-10.9)$ & $1.12(1.43-6.58)$ & 7 \\
IgE to food & 4 & 7 & - & 0.007 \\
P & 0.16 & 0.37 & & $1.90(1.68-26.6)$ \\
OR $(95 \% \mathrm{Cl})$ & $2.5(0.68-9.68)$ & $1.04(0.03-3.56)$ & 7 & - \\
IgE to aerollergens & 2 & 12 & 0.007 & $1.90(1.68-26.6)$ \\
P & 0.47 & 0.008 & 6 & 12 \\
OR $(95 \% \mathrm{Cl})$ & $0.48(0.08-2.60)$ & $6.30(1.60-24.0)$ & 0.97 & 0.02 \\
Family history of atopy & 6 & 16 & $1.0(0.59-1.59)$ & $1.68(1.22-23.3)$ \\
P & 0.58 & 0.04 & $2.66(1.05-7.00)$ & \\
OR $(95 \% \mathrm{Cl})$ & $0.65(0.21-1.57)$ & & & \\
\hline
\end{tabular}

FPIES: food protein-induced enterocolitis syndrome.

challenge was 38.2 (29-48) months. By the end of the study period, $28.5 \%$ of the patients with egg FPIES remained allergic to raw egg (Figure 2c), yet could consume baked egg. These subjects were not able to tolerate egg freely. In our study, there were no egg FPIES patients with IgE sensitisation.

\section{Long-term respiratory atopic manifestations}

During the study period ( $92 \pm 54.4$ months), 25 patients had asthma symptoms, 14 of these (56\%) had IgE sensitisation to aeroallergens during the study period (Table 2). FPIES patients with IgE sensitisation to food had a statistically significant higher risk of additional sensitisation to aeroallergens. In turn, sensitisation was positively correlated with family history of atopy and respiratory symptoms as opposed to the history of eczema. Moreover, respiratory symptoms were significantly associated with fish FPIES cases (Table 2). These findings were also confirmed by the multiple regression analysis, while probability function revealed the longitudinal effect of sensitisation on respiratory symptoms. All patients with aeroallergen sensitisation developed asthma at a much earlier time than non-sensitised patients (Figure 3a). In addition, the cumulative hazard ratio for developing asthma symptoms over time was significantly higher in patients with sensitisation to aeroallergens compared to those without sensitisation or with sensitisation to food only (Figure 3b). Moreover, the time at which tolerance was achieved, regardless of the offending food, was found to be a significant factor for the development of respiratory symptoms. The longer the allergy persistence, the higher the hazards risk (Figure $3 \mathrm{c}$ ).

\section{Discussion}

We present one of the largest European series of FPIES patients published to date from one of the major referral allergy centres of our country. The lengthy follow-up period allowed a reliable clinical assessment that included the recognition of atypical cases, confirmation of persistency or tolerance in relation to lgE sensitisation and emergence of delayed atopic symptoms.

The majority of patients with FPIES have undetectable food IgE to the suspected food. Although testing for IgE sensitisation is not recommended in routine for patients with FPIES, 2-20\% subjects were sensitised to the offending FPIES-related food. ${ }^{19-21}$ Previous studies have shown that IgE sensitisation to the offending food was associated with persistent allergy to the involved antigen, while sensitisation to other food proteins did not appear to delay tolerance acquisition. 2,14,15,22,23 This was not the case with our study as no significant correlation between IgE sensitisation to the offending food and tolerance acquisition was encountered.

Food protein-induced enterocolitis syndrome is a pathophysiologically, non-IgE-mediated disorder, yet atopic disease and a family history of allergy coexist frequently. ${ }^{19-23}$ Globally, eczema is the most common concomitant atopic disorder at diagnosis. Evaluation of eczema existence and family history of atopy varies between studies, with higher rate of existence often reported in the studies from the United States and Australia compared to those from Israel and Italy. In our study, eczema and family history of atopy in FPIES cases at baseline were compatible with data reporting lower rates. History of eczema was not significantly correlated neither to IgE sensitisation to food nor to aeroallergens. Therefore, one could assume that in our patients IgE sensitisation didn't occur through a severe impaired skin barrier.

Other atopic diseases, including asthma and allergic rhinitis, were reported in a recent study highlighting that there was no direct causation between FPIES and atopic comorbidities. $^{20}$ Similarly, in our study, comorbid atopic symptoms were found in accordance with the general population prevalence. ${ }^{24}$ However, patients with FPIES and food IgE sensitisation were two times at a higher risk of 


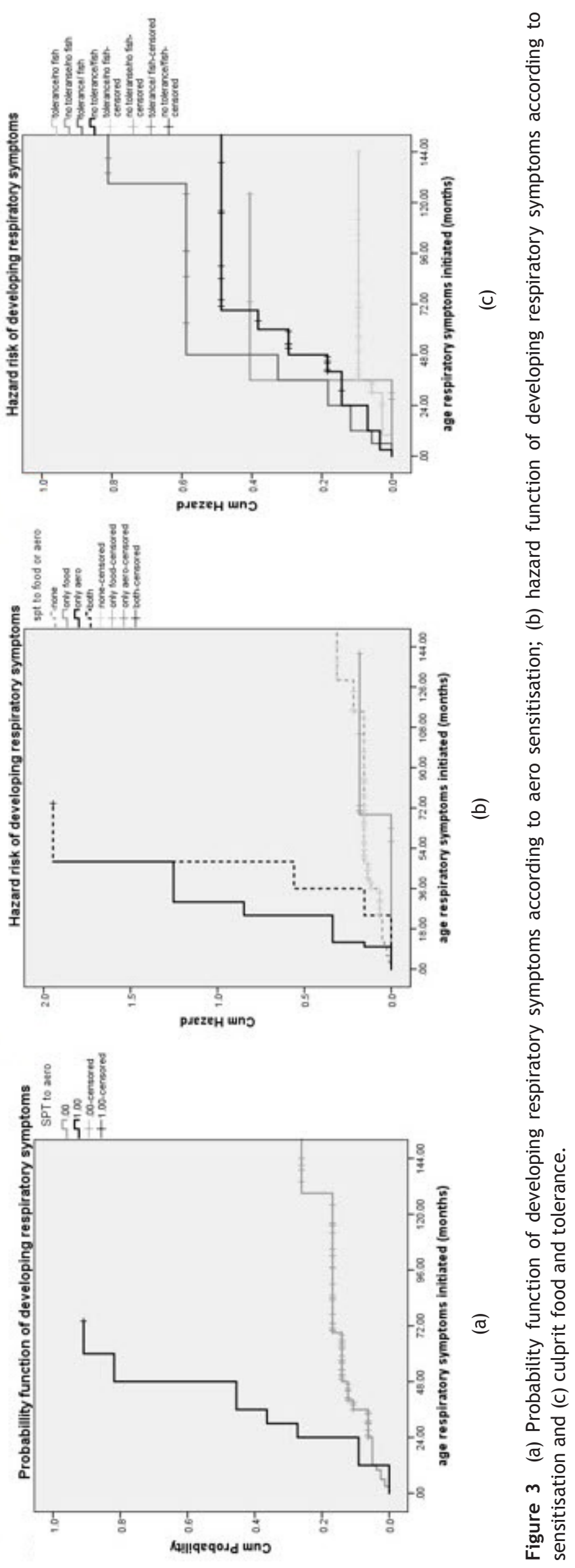


developing IgE sensitisation to aeroallergens independent of other factors. In turn, in the majority of cases, aeroallergen sensitisation was the leading factor for developing respiratory symptoms during preschool years. Conversely, food sensitisation, if not followed by aeroallergen sensitisation, did not constitute a risk factor. The above observations have been described previously in other reports, highlighting that sensitisation to food in infancy is a marker of increased risk of sensitisation to inhaled allergens and consequently asthma symptoms later in life. ${ }^{25,26}$ Therefore, based on our data, IgE sensitisation may not necessarily affect FPIES outcome but may predispose to asthma later in life.

Fish FPIES was associated with an increased hazard of developing sensitisation to aeroallergens and asthma during infancy. An explanation for this might be the fact that the majority of our FPIES patients were fish-driven; this possibly might have biased our results. On the other hand, tolerance to fish FPIES, if ever achieved, is generally delayed, driving inflammatory molecular mechanisms towards an IgE switching. We believe that the emergence of an IgE-allergic mechanism is not specific to the allergen but rather driven by the persistent allergic response. This was supported by the fact that the delay or absence of tolerance was also related to an increased hazard of developing aeroallergen sensitisation and subsequent respiratory symptoms regardless of the offending food. In a recent report by Meyer et al., the authors proposed an integrated non-lgE- and IgEmediated allergic march. In this model, we observe that a delayed-resolving FPIES coincides with the emergence of an IgE comorbidity, namely rhinitis and asthma. ${ }^{19}$ We propose an explanatory underlying mechanism whereby the persistent non-lgE allergy in predisposed individuals results in increased production of TNF $\alpha$, disrupted epithelial barrier and subsequent sensitisation with aeroallergens with ultimate development of rhinitis and asthma.

In our study, fish was the most commonly reported triggering food. Similar results have been reported previously in Mediterranean countries ${ }^{14,22,27,28}$ in contrast to the data from the United States and Sweden. ${ }^{22,28}$ Concerning the type of offending fish, our finding that five subjects tolerated a type of fish other than the culprit one, has strengthened previous data from Italy. ${ }^{30}$ The immunopathology behind these observations remains foggy. Perhaps, there are species-specific parvalbumin epitopes associated with an increase in TNF- $\alpha$ and human leukocyte antigen-DR isotype (HLA-DR) marker. ${ }^{31}$

Cow milk was the second most common triggering food in our study, although it is the most commonly reported food throughout the world. ${ }^{1-4,23,28}$ Failure to thrive was the main clinical finding in a minority of subjects, most likely because of prompt diagnosis. Thus, awareness of the disease and early diagnosis ensured adequate infantile growth in chronic FPIES cases. Notably, two patients were admitted due to urticaria after milk consumption, yet non-lgE sensitisation was found. Urticaria is primarily an IgE-mediated disease; however, it occasionally occurs through non-lgEmediated mechanisms, namely by degranulation of mast cells and basophils with subsequent histamine and release of other inflammatory mediators. ${ }^{32}$ Therefore, cell-mediated mechanisms shared in common with both urticaria and FPIES may explain the coexistence of both entities.
Egg was the next in frequency of culprit foods recorded. One egg FPIES case was caused exclusively by egg yolk. Difference in the antigenicity resulting in FPIES symptoms between egg white and egg yolk has been described previously in few cases. ${ }^{33}$ Chicken serum albumin (Gal d 5) and YGP42 (Gal d 6) were suggested to be the major allergens of IgE-mediated egg yolk allergy. ${ }^{34,35}$ However, the components of egg yolk inducing gastrointestinal symptoms in FPIES cases remain to be elucidated.

The median age of tolerance acquisition varied according to the offending food and paralleled the age of onset. Resolution of milk FPIES was achieved at a younger age, followed by egg. Tolerance to fish was clearly delayed, although almost half of fish FPIES and one-third egg FPIES subjects had not outgrown FPIES until the end of the study period. Remarkably, as reported previously, fish FPIES presented with severe clinical manifestations, a fact that may have delayed or restrained patients from proceeding to OFC..$^{31,36}$ In addition, a previous study showed that the consumption of fish by Greek children was relatively low, implying that fish was not widely considered an important nutritive food. ${ }^{37}$ These two points might explain the fact that few fish cases were lost to follow-up or refused OFC.

It is worth mentioning that there were some FPIES cases that reacted differently when the offending food was cooked or modified. Three cow's milk FPIES patients reacted to raw milk but were able to consume yogurt or cheese. Even though none of these patients had an IgE allergy profile, a similar phenomenon was reported in patients with a specific IgE against $\beta$-lactoglobulin gastrointestinal phenotype. These patients also benefited from a diet with other dairy products. ${ }^{38}$ Moreover, two egg FPIES patients could consume baked egg. Tolerance to small amount of cooked offending food that has been described recently could be due to the fact that FPIES reaction is dose-dependent. ${ }^{39}$ Yet, degradation of responsible conformational epitope could help some FPIES phenotypes tolerate the offending food if cooked well. Alternatively, the glycosylation of ovalbumin with monosaccharides (i.e. mannose) affected differently specific dendritic cell receptors integration, MHC antigen presentation and antigen-specific $\mathrm{T}$ cell activation, resulting in an attenuated allergic response to egg allergy. ${ }^{40}$

Based on the described endoscopic and immunological findings in infants with FPIES, several underlying potential mechanisms have been suggested ${ }^{8-13,17,18}$ Trying to incorporate various immunological interactions and clinical presentations involved in the pathophysiology of FPIES, a potential schematic illustration is depicted in Figure 4. Although the cell ultimately responsible for antigen recognition and initiation of reaction in FPIES remains unknown, it is recognised that children presenting with FPIES produce high levels of TNF- $\alpha$ and have substantially low levels of TGF- $\beta$. Therefore, in acute FPIES, it is believed that a form of antigen presentation activates Th1 cells producing TNF$\alpha$. In the absence of the regulatory role of TGF- $\beta$, a local inflammatory reaction takes place with the accumulation and activation of neutrophils and eosinophils with enhancement of their cytotoxicity. TNF- $\alpha$ also acts on the epithelium, increasing its permeability and altering the intestinal tight junction barrier. Besides, TNF- $\alpha$ directly acts on the 


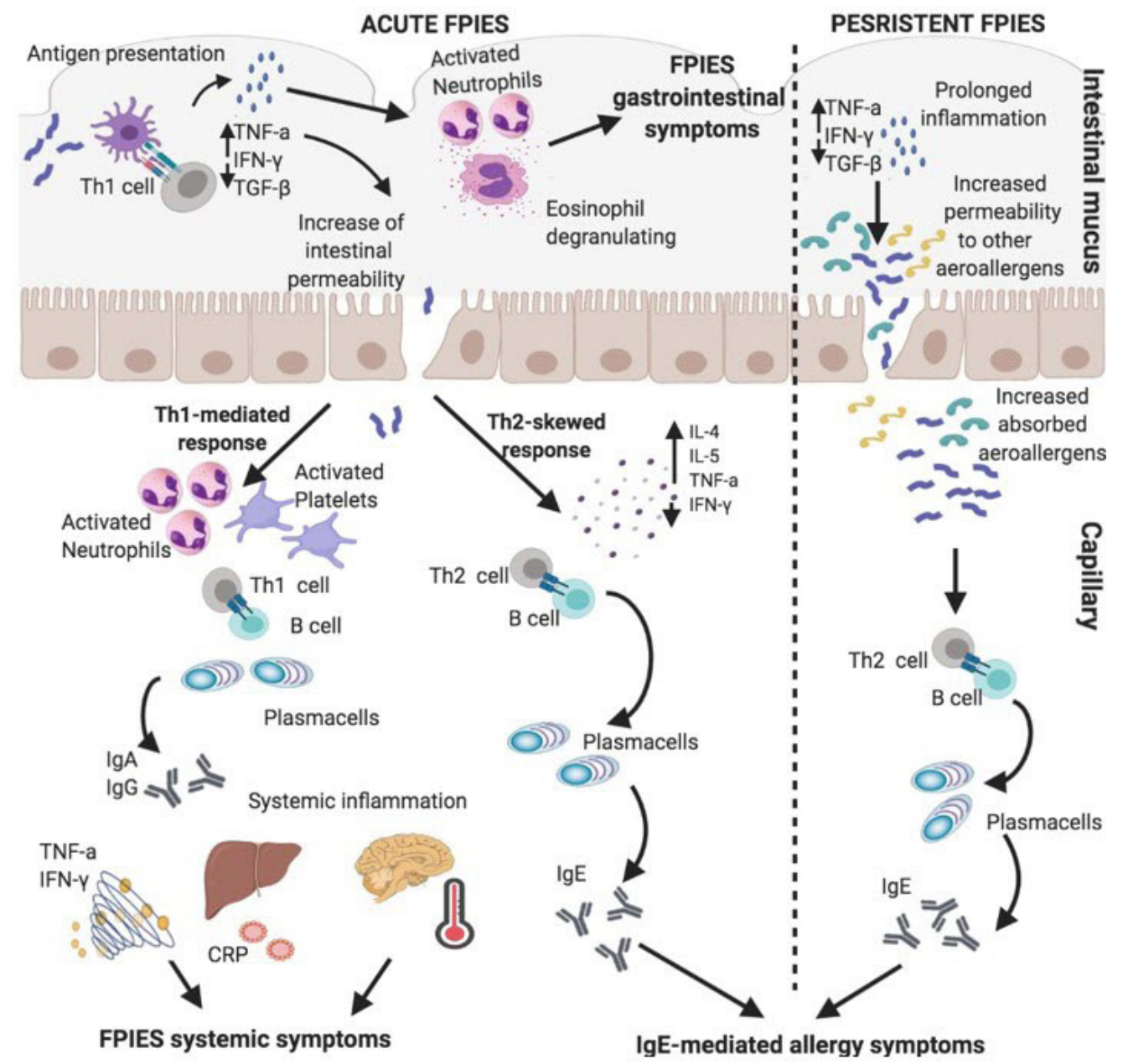

Figure 4 A schematic representation of the possible immunological pathways resulting in food protein-induced enterocolitis syndrome's (FPIES) clinical symptoms and Th2 skewing. Following antigen presentation, in the absence of a regulatory role of TGF- $\beta$, TNF- $\alpha$ alters the intestinal tight junction barrier. As a result, a local cytotoxic inflammatory response is produced with profound local enhancement of neutrophils and eosinophils. Systemic action of TNF- $\alpha$ on the liver induces the production of acutephase proteins, and on the hypothalamus increases body temperature. In distinct cases, a Th2-skewed response may be involved implying an increase in IL-4 and IL-5 and a decrease in IFN- $\gamma$ expression by peripheral T cells. This consecutively switches B cells in predisposed individuals towards the production of specific IgE to food and/or aeroallergens, eventually leading to a classic IgEmediated reaction. Additionally, in persistent FPIES cases (i.e. fish or egg), the above-mentioned prolonged immune deviation leads to persistent inflammation of the intestinal mucosa, thereby increasing its permeability to aeroallergens predisposing to respiratory atopic symptom.

hypothalamus, increasing the body temperature, and the liver, promoting the production of acute-phase proteins (i.e. C-reactive protein [CRP]), resulting in systemic inflammation. The inflammatory process is further enhanced by the absence of the regulatory role of TGF- $\beta$. In distinct cases, a Th2-skewed response may be involved, implying an increase in IL- 4 and IL-5 and a decrease in IFN- $\gamma$ expression by peripheral T cells. ${ }^{13}$ This consecutively switches B cells, in predisposed individuals, towards the production of specific IgE to food and/or aeroallergens, eventually leading to a classic IgE-mediated reaction. Additionally, in persistent FPIES cases (i.e. fish or egg), the prolonged immune-deviation described above leads to persistent inflammation of the intestinal mucosa, increasing its permeability to aeroallergens, predisposing to respiratory atopic symptoms (Figure 4).
Certain limitations have to be taken into consideration. The narrow range of offending foods in the present study might not be a representative of the whole allergens responsible in the community. Moreover, as egg was the culprit allergen in a minority of our patients, safe conclusions cannot be drawn concerning the long-term outcome in relation to IgE sensitisation. In addition, the precise time of tolerance cannot be reliably determined as it was bounded by predefined time intervals of prospective evaluation. Even though FPIES cases were prospectively and consistently recorded by specialised personnel for a lengthy time period, higher numbers of IgE FPIES cases are definitely needed to extrapolate robust correlations. However, the main results were repeatedly confirmed by many statistical approaches, enhancing the reliability of our findings. 


\section{Conclusion}

In the majority of FPIES subjects presented with acute form of the disease, the most common offending food in our Greek cohort was fish. Food IgE sensitisation was not the cause of an underlying impaired skin barrier and did not affect tolerance but was rather linked to IgE aeroallergens sensitisation, which predisposed to asthma. Moreover, delay in tolerance acquisition may also lead to prolonged and consistent Th2 immune activation, predisposed to respiratory symptoms. Larger population studies and longer follow-up periods and assessments of the underlying immunological mechanisms are required to shed light on the relation between FPIES, atopy and IgE sensitisation.

\section{Acknowledgements}

We thank Dr Fani Giannoula for children's management, Cathy Lazarou for English editing, the nurses of our department for their valuable help and all parents and children who volunteered to participate in our study.

\section{Conflict of interest}

None.

\section{Author contributions}

All authors have contributed substantially to the conception and designing of the work, or the acquisition, analysis, and interpretation of data for the work and drafting the work or revising it critically for important intellectual content. They all have approved the final version to be published and agreed to be accountable for all aspects of the work in ensuring that questions related to the accuracy or integrity of any part of the work are appropriately investigated and resolved.

\section{Funding}

This research did not receive any specific grant from funding agencies in the public, commercial, or not-for-profit sectors.

\section{References}

1. Leonard SA, Pecora V, Fiocchi AG, Nowak-Wegrzyn A. Foodprotein-induced enterocolitis syndrome: A review of the new guidelines. World Allergy Organ J. 2018;11:4. doi: 10.1186/ s40413-017-0182-z.

2. Nowak-Węgrzyn A, Chehade M, Groetch ME, Spergel JM, Wood RA, Allen K, et al. International consensus guidelines for the diagnosis and management of food protein-induced enterocolitis syndrome: Executive summary-Workgroup report of the Adverse Reactions to Foods Committee, American Academy of Allergy, Asthma \& Immunology. J Allergy Clin Immunol. 2017;139:1111-26.e4. doi: 10.1016/j.jaci.2016.12.966.
3. Sicherer SH, Eigenmann PA, Sampson HA. Clinical features of food protein-induced enterocolitis syndrome. J Pediatr. 1998;133(2): 214-9. doi: 10.1016/s0022-3476(98)70222-7. PMID: 9709708.

4. Cherian S, Varshney P. Food protein-induced enterocolitis syndrome (FPIES): Review of recent guidelines. Curr Allergy Asthma Rep. 2018;18:28. doi: 10.1007/s11882-018-0767-9. PMID: 29623454.

5. Mehr S, Campbell DE. Food protein-induced enterocolitis syndrome: Guidelines summary and practice recommendations. Med J Aust. 2019;210:94-9. doi: 10.5694/mja2.12071. PMID: 30656696.

6. Miceli Sopo S, Fantacci C, Bersani G, Romano A, Liotti L, Monaco $S$. Is food protein-induced enterocolitis syndrome only a non-IgE-mediated food allergy? Allergol Immunopathol (Madr). 2018;46:499-502. doi: 10.1016/j.aller.2017.10.004. PMID: 29472022.

7. Lee E, Campbell DE, Barnes EH, Mehr SS. Resolution of acute food protein-induced enterocolitis syndrome in children. J Allergy Clin Immunol Pract. 2017;5:486-8.e1. doi: 10.1016/j. jaip.2016.09.032.

8. Goswami R, Blazquez AB, Kosoy R, Rahman A, NowakWęgrzyn A, Berin MC. Systemic innate immune activation in food protein-induced enterocolitis syndrome. J Allergy Clin Immunol. 2017;139:1885-96.e9. doi: 10.1016/j.jaci.2016.12.971.

9. Konstantinou GN, Bencharitiwong R, Grishin A, Caubet JC, Bardina $\mathrm{L}$, Sicherer $\mathrm{SH}$, et al. The role of casein-specific IgA and TGF- $\beta$ in children with food protein-induced enterocolitis syndrome to milk. Pediatr Allergy Immunol. 2014;25:651-6. doi: 10.1111/pai.12288.

10. Caubet JC, Nowak-Węgrzyn A. Current understanding of the immune mechanisms of food protein-induced enterocolitis syndrome. Expert Rev Clin Immunol. 2011;7:317-27. PMID: 21595598.

11. Caubet JC, Bencharitiwong R, Ross A, Sampson HA, Berin MC, Nowak Węgrzyn A. Humoral and cellular responses to casein in food protein-induced enterocolitis to cow's milk. J Allergy Clin Immunol. 2017;139:572-83. doi: 10.1016/j.jaci.2016.02.047.

12. Adel-Patient K, Lezmi G, Castelli FA, Blanc S, Bernard H, Soulaines $P$, et al. Deep analysis of immune response and metabolic signature in children with food protein-induced enterocolitis to cow's milk. Clin Transl Allergy. 2018;8:38. doi: 10.1186/s13601-018-0224-9.

13. Mori F, Barni S, Cianferoni A, Pucci N, de Martino M, Novembre E. Cytokine expression in CD3+ cells in an infant with food protein-induced enterocolitis syndrome (FPIES): Case report. Clin Dev Immunol. 2009;2009:679381. PMID: 20011655.

14. Katz Y, Goldberg MR, Rajuan N, Cohen A, Leshno M. The prevalence and natural course of food protein-induced enterocolitis syndrome to cow's milk: A large-scale, prospective population-based study. J Allergy Clin Immunol. 2011;127:64753. doi: 10.1016/j.jaci.2010.12.1105.

15. Douros K, Tsabouri S, Feketea G, Grammeniatis V, Koliofoti EG, Papadopoulos M, et al. Retrospective study identified fish and milk as the main culprits in cases of food protein-induced enterocolitis syndrome. Acta Paediatr. 2019;108:1901-04. doi: 10.1111/apa.14779.

16. Caubet JC, Ford LS, Sickles L, Järvinen KM, Sicherer SH, Sampson HA, et al. Clinical features and resolution of food protein-induced enterocolitis syndrome: 10-year experience. J Allergy Clin Immunol. 2014;134:382-9. doi: 10.1016/j. jaci.2014.04.008.

17. Morita H, Nomura I, Orihara K, Yoshida K, Akasawa A, Tachimoto $\mathrm{H}$, et al. Antigen-specific T-cell responses in patients with non-lgE-mediated gastrointestinal food allergy are predominantly skewed to $\mathrm{T}(\mathrm{H}) 2$. J Allergy Clin Immunol. 2013;131:590-2, e1-6. doi: 10.1016/j.jaci.2012.09.005.

18. Ishige $T$, Yagi H, Tatsuki M, Hatori R, Nishida Y, Takizawa T, et al. Endoscopic findings in the acute phase of food protein-induced enterocolitis syndrome. Pediatr Allergy Immunol. 2015;26:90-1. PMID: 25534006. 
19. Meyer R, Fox AT, Chebar Lozinsky A, Michaelis LJ, Shah N. Non-IgE-mediated gastrointestinal allergies - Do they have a place in a new model of the allergic march. Pediatr Allergy Immunol. 2019;30:149-58. doi: 10.1111/pai.13000.

20. Ruffner MA, Wang KY, Dudley JW, Cianferoni A, Grundmeier RW, Spergel JM, et al. Elevated atopic comorbidity in patients with food protein-induced enterocolitis. J Allergy Clin Immunol Pract. 2020;8:1039-46. doi: 10.1016/j.jaip.2019.10.047

21. Baker MG, Nowak-Wegrzyn A. Food protein-induced enterocolitis syndrome: Epidemiology and comorbidities. Curr Opin Allergy Clin Immunol. 2020;20:168-74. doi: 10.1097/ACI. 0000000000000615.

22. Vila L, Garcia V, Rial MJ, Novoa E, Cacharron T. Fish is a major trigger of solid food protein-induced enterocolitis syndrome in Spanish children. J Allergy Clin Immunol Pract. 2015;3:621-3. doi: 10.1016/j.jaip.2015.03.006.

23. Nowak-Wegrzyn A, Warren CM, Brown-Whitehorn $T$, Cianferoni A, Schultz-Matney F, Gupta RS. Food protein-induced enterocolitis syndrome in the US population-based study. J Allergy Clin Immunol. 2019;144:1128-30. PMID: 31288044.

24. Papadopoulou A, Hatziagorou E, Matziou VN, Grigoropoulou DD, Panagiotakos DB, Tsanakas JN, et al. Comparison in asthma and allergy prevalence in the two major cities in Greece: The ISAAC phase II survey. Allergol Immunopathol (Madr). 2011;39:347-55. PMID: 21342745.

25. Priftis KN, Mermiri D, Papadopoulou A, Papadopoulos M, Fretzayas A, LagonaE. Asthma symptoms and bronchial reactivity in school children sensitized to food allergens in infancy. J Asthma. 2008;45:590-5. doi: 10.1080/02770900802032941.

26. Hose AJ, Depner M, Illi S, Lau S, Keil T, Wahn U, et al. MAS, PASTURE study groups. Latent class analysis reveals clinically relevant atopy phenotypes in 2 birth cohorts. J Allergy Clin Immunol. 2017;139:1935-45. e12. doi: 10.1016/j. jaci.2016.08.046.

27. Miceli Sopo S, Giorgio V, Dellolacono I, Novembre E, Mori F, Onesimo R. A multicentre retrospective study of 66 Italian children with food protein-induced enterocolitis syndrome: Different management for different phenotypes. Clin Exp Allergy. 2012;42:1257-65. doi: 10.1111/j.1365-2222. 2012.04027.x.

28. Xepapadaki P, Kitsioulis NA, Manousakis E, Manolaraki I, Douladiris N, Papadopoulos NG. Remission patterns of food protein-induced enterocolitis syndrome in a Greek pediatric population. Int Arch Allergy Immunol. 2019;180:113-9. doi: 10.1159/000500860.

29. Karefylaki S, Gustafsson D. Recovery from food protein-induced enterocolitis syndrome caused by fish. Pediatr Allergy Immunol. 2016;27:105-6. doi: 10.1111/pai.12488.
30. Miceli Sopo S, Monaco S, Badina L, Barni S, Longo G, Novembre E, et al. Food protein-induced enterocolitis syndrome caused by fish and/or shellfish in Italy. Pediatr Allergy Immunol. 2015;26:731-6. PMID: 26287446.

31. Gonzalez-Delgado P, Caparros E, Moreno MV, Clemente F, Flores E, Velasquez L, et al. Clinical and immunological characteristics of a pediatric population with food protein-induced enterocolitis syndrome (FPIES) to fish. Pediatr Allergy Immunol. 2016;27:269-75. doi: 10.1111/pai.12529.

32. Radonjic-Hoesli S, Hofmeier KS, Micaletto S, SchmidGrendelmeier P, Bircher A, Simon D. Urticaria and angioedema: An Update on Classification and Pathogenesis. Clin Rev Allergy Immunol. 2018;54:88-101. doi: 10.1007/s12016-017-8628-1.

33. Shimomura M, Tanaka H, Meguro T, Kimura M. Three cases of food protein-induced enterocolitis syndrome caused by egg yolk. Allergol Int. 2019;68:110-1. PMID: 29759658.

34. Dhanapala P, De Silva C, Doran T, Suphioglu C. Cracking the egg: an insight into egg hypersensitivity. Mol Immunol. 2015;66:375-83. PMID: 25965316.

35. De Silva C, Dhanapala P, King S, Doran T, Tang M, Suphioglu C. Immunological comparison of native and recombinant hen's egg yolk allergen, chicken serum albumin (Gal d 5), produced in Kluveromyceslactis. Nutrients. 2018;10. pii: E757. PMID: 29895783.

36. Ruiz-García M, Díez CE, García SS, del Río PR, Ibáñez MD. Diagnosis and natural history of food-protein induced enterocolitis syndrome in children from a tertiary hospital in central Spain. J Investig Allergol Clin Immunol. 2014;24:354-6. PMID: 25345307.

37. Papadopoulou A, Panagiotakos DB, Hatziagorou E, Antonogeorgos G, Matziou VN, Tsanakas JN, et al. Antioxidant foods consumption and childhood asthma and other allergic diseases: The Greek cohorts of the ISAACII survey. Allergol Immunopathol (Madr). 2015;4:353-60. doi: 10.1016/j. aller.2014.03.002.

38. Poza-Guedes P, Barrios Y, González-Pérez R, Sánchez-Machínl, Franco A, Matheu V. Role of specific IgE to $\beta$-lactoglobulin in the gastrointestinal phenotype of cow's milk allergy. Allergy Asthma Clin Immunol 2016;12:7. PMID: 26909103.

39. Rupa P, Mine Y. Comparison of glycated ovalbumin-monosaccharides in the attenuation of ovalbumin-induced allergic response in a BALB/C mouse model. J Agric Food Chem. 2019;67:8138-48. PMID: 31294563.

40. Miceli Sopo S, Buonsenso D, Monaco S, Crocco S, Longo G, Calvani M. Food protein-induced enterocolitis syndrome (FPIES) and well-cooked foods: A working hypothesis. Allergol Immunopathol (Madr). 2013;41:346-8. doi: 10.1016/j. aller.2012.06.008. 